\title{
A Rational Protocol for the Synthesis of Arylated
}

\section{Bipyridine Ligands via a Cycloaddition Pathway}

\author{
Stéphane Diring, ${ }^{a}$ Pascal Retailleau ${ }^{b}$ and Raymond Ziessel $^{a, *}$
}

\section{Supporting Information (17 pages)}

\section{Table of Contents}

Pages

$\begin{array}{ll}\text { General Methods } & \text { S1 }\end{array}$

$\begin{array}{ll}\text { Electrochemical Measurements. } & \text { S2 }\end{array}$

$\begin{array}{ll}\text { Spectroscopic Measurements. } & \text { S2 }\end{array}$

Experimental details of compounds $3,4,5,6,8,9,12,13,14,15,21,22,25 . \quad S 3$

X-Ray data for compounds 2a, 2b, 13, 23, 24, $26 . \quad$ S15

Spectroscopic Data. of compounds 9, 10, 12a, 12b, 14, 22, Figure S1 S16

Solvent Effects for $4 \mathbf{a} ; \mathbf{4 b} ; \mathbf{5 a} ; \mathbf{5 b}$, Figure S2 $\quad$ S17

$\begin{array}{ll}\text { References supporting information. } & \text { S18 }\end{array}$

General Methods. 200.1 $\left({ }^{1} \mathrm{H}\right), 300.1\left({ }^{1} \mathrm{H}\right), 400\left({ }^{1} \mathrm{H}\right), 50.3\left({ }^{13} \mathrm{C}\right), 75.46\left({ }^{13} \mathrm{C}\right)$ and $100.3\left({ }^{13} \mathrm{C}\right)$ MHz NMR spectra were recorded at room temperature using the residual proton resonances in deuterated solvents as internal references. Fast-atom bombardment mass spectra were obtained using a ZAB-HF-VB-analytical apparatus in positive mode with $m$ - 
nitrobenzylalcohol $(m$-NBA) as matrix. FT-IR spectra were recorded on the neat liquids or as thin films, prepared with a drop of dichloromethane solution evaporated to dryness on $\mathrm{KBr}$ pellets. Chromatographic purification was conducted using either standardized aluminium oxide 90 or silica gel Si $60(40-63 \mu \mathrm{m})$. Thin Layer Chromatography (TLC) was performed on aluminium oxide or silica gel plates coated with fluorescent indicator. All mixtures of solvents are given in $\mathrm{v} / \mathrm{v}$ ratio.

\section{Electrochemical Measurements.}

Electrochemical studies employed cyclic voltametry with a conventional 3-electrode system using a voltammetric analyser equipped with a Pt microdisk $\left(2 \mathrm{~m}^{2}\right)$ working electrode and a silver wire counter-electrode. Ferrocene was used as an internal standard and was calibrated against a saturated calomel reference electrode (SCE) separated from the electrolysis cell by a glass frit presoaked with electrolyte solution. Solutions contained the electro-active substrate in deoxygenated and anhydrous dichloromethane containing doubly recrystallized tetra-nbutylammonium hexafluorophosphate $(0.1 \mathrm{M})$ as supporting electrolyte. The quoted halfwave potentials were reproducible within $\approx 10 \mathrm{mV}$.

Spectroscopic Measurements. The electronic spectra were measured under ambient conditions and solvent for spectroscopy were used as received and recorded on an absorption spectrometer. The steady-state fluorescence emission and excitation spectra were obtained by using a spectrofluorimeter instrument. All fluorescence spectra were corrected. The fluorescence quantum yield ( $\left.\Phi_{\mathrm{exp}}\right)$ was calculated from eq $1 .{ }^{\mathrm{S} 1}$

$$
\Phi_{\text {exp }}=\Phi_{r e f} \frac{F\left\{1-\exp \left(-A_{r e f} \ln 10\right)\right\} n^{2}}{F_{r e f}\{1-\exp (-A \ln 10)\} n_{r e f}^{2}}
$$


Here, $F$ denotes the integral of the corrected fluorescence spectrum, $A$ is the absorbance at the excitation wavelength, and $n$ and $n_{\text {ref }}$ are respectively the measured refractive indexes of dichloromethane $(\mathrm{n}=1.4224)$ and $1 \mathrm{~N} \mathrm{H}_{2} \mathrm{SO}_{4}\left(\mathrm{n}_{\mathrm{ref}}=1.3395\right)$ at $296 \mathrm{~K}$.

The reference system used was quinine bisulfate dihydrate $\left(\Phi_{\mathrm{ref}}=0.546, \lambda_{\mathrm{exc}}=366 \mathrm{~nm}\right)^{\mathrm{S} 2}$ in $1 \mathrm{~N} \mathrm{H}_{2} \mathrm{SO}_{4}$ solution. Luminescence lifetime were measured on a time-resolved spectrofluorimeter, using Time-Correlated Single Photon Mode coupled to a Stroboscopic system. The excitation source was a thyratron-gated flash lamp filled with nitrogen gas. No filter was used for the excitation. The instrument response function was determined by using a light-scattering solution.

Materials. $\mathrm{CH}_{2} \mathrm{Cl}_{2}$ was distilled from $\mathrm{P}_{2} \mathrm{O}_{5}$, 2-cyanopyrydine, hydrazine hydrate, 2-phenyl glyoxal, 4-ethynyltoluene, 3,3-dimethylbut-1-yne, N,N-dimethylprop-2-yn-1-amine, $\mathrm{K}_{2} \mathrm{PtCl}_{4}$, CuI, THF, o-dichlorobenzene, ${ }^{\mathrm{i}} \mathrm{Pr}_{2} \mathrm{NH}$, TEA, piperidine were used as purchased. $\left[\mathrm{Pd}\left(\mathrm{PPh}_{3}\right)_{2} \mathrm{Cl}_{2}\right]^{\mathrm{S} 3},\left[\mathrm{Pd}\left(\mathrm{PPh}_{3}\right)_{4}\right] . \mathrm{S} 4$

3-(3,4,5-tris(dodecyloxy)phenyl)-6-phenyl-2,2'-bipyridine (3a). Prepared using procedure 1; from 5-phenyl-3-(2-pyridyl)-as-triazine (110 mg, $0.46 \mathrm{mmol})$, 3,4,5-tris(dodecyloxy)-5ethynylbenzene $(300 \mathrm{mg}, 0.46 \mathrm{mmol})$ and 1,2-dichlorbenzene $(1 \mathrm{ml})$; chromatography on silica gel eluting with dichloromethane - petroleum ether $(v / v 30 / 70)$ to dichloromethane to give $186 \mathrm{mg}(47 \%)$ of $\mathbf{3 a}$ as a white solid after reprecipitation in a dichloromethane methanol mixture; ${ }^{1} \mathrm{H} \mathrm{NMR}\left(\mathrm{CDCl}_{3}, 400 \mathrm{MHz}\right): \delta 8.58\left(\mathrm{dt}, 1 \mathrm{H},{ }^{3} \mathrm{~J}=5.0 \mathrm{~Hz},{ }^{4} \mathrm{~J}=1 \mathrm{~Hz}\right), 8.10$ $\left(\mathrm{d}, 2 \mathrm{H},{ }^{3} J=7.0 \mathrm{~Hz}\right), 7.85\left(\right.$ ABsys, $\left.2 \mathrm{H}, J_{\mathrm{AB}}=8.0 \mathrm{~Hz}, v_{0} \delta=21.1 \mathrm{~Hz}\right), 7.62\left(\mathrm{td}, 1 \mathrm{H},{ }^{3} J=7.5 \mathrm{~Hz}\right.$, $\left.{ }^{4} J=2.0 \mathrm{~Hz}\right), 7.49-7.39(\mathrm{~m}, 4 \mathrm{H}), 7.18(\mathrm{ddd}, 1 \mathrm{H}, J=7.5 \mathrm{~Hz}, 4.9 \mathrm{~Hz}, 1.3 \mathrm{~Hz}), 6.35$ (s, 2H), $3.93\left(\mathrm{t}, 2 \mathrm{H},{ }^{3} J=6.5 \mathrm{~Hz}\right), 3.74\left(\mathrm{t}, 4 \mathrm{H},{ }^{3} J=6.5 \mathrm{~Hz}\right), 1.76-1.65(\mathrm{~m}, 6 \mathrm{H}), 1.48-1.27(\mathrm{~m}, 54 \mathrm{H})$, $0.88\left(\mathrm{t}, 9 \mathrm{H},{ }^{3} \mathrm{~J}=7.0 \mathrm{~Hz}\right) ;{ }^{13} \mathrm{C} \mathrm{NMR}\left(\mathrm{CDCl}_{3}, 100 \mathrm{MHz}\right): \delta 159.2,156.0,155.7,153.0,149.2$, 
$139.1,138.9,137.6,136.1,135.3,134.3,129.1,128.8,127.3,125.0,122.4,119.9,108.3,73.5$, 69.1, 32.1, 29.9, 29.85, 29.8, 29.76, 29.5, 26.3, 26.2, 22.8, 14.2; UV-Vis $\left(\mathrm{CH}_{2} \mathrm{Cl}_{2}\right) \lambda \mathrm{nm}\left(\varepsilon, \mathrm{M}^{-}\right.$ $\left.{ }^{1} \mathrm{~cm}^{-1}\right)=306$ (21000), 274 (19300); IR (KBr, cm-1) v = $3035(\mathrm{w}), 2918(\mathrm{~s}), 2851$ (s), 1579 (s), $1456(\mathrm{~s}), 1250(\mathrm{~m}), 1090$ (s), 754 (s), $\mathrm{FAB}^{+} \mathrm{m} / \mathrm{z}$ (nature of the peak, relative intensity) $861.4\left([\mathrm{M}+\mathrm{H}]^{+}, 100\right)$; Anal. Calcd for : $\mathrm{C}_{58} \mathrm{H}_{88} \mathrm{~N}_{2} \mathrm{O}_{3}: \mathrm{C}, 80.88 ; \mathrm{H}, 10.30 ; \mathrm{N}, 3.25$; Found: C, $80.72 ; \mathrm{H}, 10.19 ; \mathrm{N}, 3.17$.

4-(3,4,5-tris(dodecyloxy)phenyl)-6-phenyl-2,2'-bipyridine (3b). Minor isomer: $70 \mathrm{mg}$ (18 \%) of $\mathbf{3 b}$ as a white solid after reprecipitation in a dichloromethane - methanol mixture; ${ }^{1} \mathrm{H}$ NMR $\left(\mathrm{CDCl}_{3}, 300 \mathrm{MHz}\right): \delta 8.74-8.68(\mathrm{~m}, 2 \mathrm{H}), 8.57\left(\mathrm{~d}, 1 \mathrm{H},{ }^{4} \mathrm{~J}=1.3 \mathrm{~Hz}\right), 8.22-8.20(\mathrm{~m}$, 2H), $7.91\left(\mathrm{~d}, 1 \mathrm{H},{ }^{4} J=1.5 \mathrm{~Hz}\right), 7.87\left(\mathrm{td}, 1 \mathrm{H},{ }^{3} J=7.8 \mathrm{~Hz},{ }^{4} J=1.8 \mathrm{~Hz}\right), 7.57-7.44(\mathrm{~m}, 3 \mathrm{H})$, $7.34(\mathrm{ddd}, 1 \mathrm{H}, J=7.5 \mathrm{~Hz}, 4.8 \mathrm{~Hz}, 1.1 \mathrm{~Hz}), 6.97(\mathrm{~s}, 2 \mathrm{H}), 4.12-4.02(\mathrm{~m}, 6 \mathrm{H}), 1.91-1.75$ (m, $6 \mathrm{H}), 1.52-1.27(\mathrm{~m}, 54 \mathrm{H}), 0.91-0.86(\mathrm{~m}, 9 \mathrm{H}) ;{ }^{13} \mathrm{C} \mathrm{NMR}\left(\mathrm{CDCl}_{3}, 75 \mathrm{MHz}\right): \delta 157.2,156.6$, $156.3,153.8,150.8,149.1,139.7,139.5,137.0,134.3,129.2,128.9,127.2,123.9,121.7$, $118.7,117.6,106.3,73.7,69.6,32.1,32.0,30.5,29.9,29.8,29.7,29.6,29.5,26.3,22.8,14.2$; UV-Vis $\left(\mathrm{CH}_{2} \mathrm{Cl}_{2}\right) \lambda \mathrm{nm}\left(\varepsilon, \mathrm{M}^{-1} \mathrm{~cm}^{-1}\right)=306 \mathrm{sh}$ (18600), 285 (29500), 267 (26200); IR ( $\mathrm{KBr}$, cm-1) $v=2955$ (m), 2916 (s), 2849 (s), $1584(\mathrm{~m}), 1504$ (m), 1468 (m), 1240 (m), 1115 (s), $774(\mathrm{~m}) ; \mathrm{FAB}^{+} \mathrm{m} / z$ (nature of the peak, relative intensity) $861.4\left([\mathrm{M}+\mathrm{H}]^{+}, 100\right)$; Anal. Calcd for: $\mathrm{C}_{58} \mathrm{H}_{88} \mathrm{~N}_{2} \mathrm{O}_{3}$ : C, 80.88; H, 10.30; N, 3.25; Found: C, 80.67; H, 10.12; N, 3.13.

3-(9,9-dimethylfluoren-2-yl)-6-phenyl-2,2'-bipyridine (4a). Prepared using procedure 1; from 5-phenyl-3-(2-pyridyl)-as-triazine (108 mg, $0.46 \mathrm{mmol})$, 2-ethynyl-9,9-dimethylfluorene $(100 \mathrm{mg}, 0.46 \mathrm{mmol})$ and 1,2-dichlorobenzene $(1 \mathrm{ml})$; chromatography on silica gel eluting with dichloromethane to dichloromethane - methanol ( $v / v 99 / 1)$ to give $65 \mathrm{mg}(33 \%)$ of $\mathbf{4 a}$ as a white to light yellow powder; ${ }^{1} \mathrm{H} \mathrm{NMR}\left(\mathrm{CDCl}_{3}, 300 \mathrm{MHz}\right): \delta 8.52$ (ddd, $1 \mathrm{H}, J=4.8 \mathrm{~Hz}, 1.6$ 
$\mathrm{Hz}, 1.0 \mathrm{~Hz}), 8.16\left(\mathrm{~d}, 2 \mathrm{H},{ }^{3} J=7.0 \mathrm{~Hz}\right), 7.92$ (ABsys, $\left.2 \mathrm{H}, J_{\mathrm{AB}}=8.1 \mathrm{~Hz}, \mathrm{v}_{0} \delta=26.3 \mathrm{~Hz}\right), 7.74-$ $7.29(\mathrm{~m}, 11 \mathrm{H}), 7.17$ (ddd, 1H, $J=7.0 \mathrm{~Hz}, 5.0 \mathrm{~Hz}, 1.9 \mathrm{~Hz}), 7.06\left(\mathrm{~d}, 1 \mathrm{H},{ }^{4} J=1.1 \mathrm{~Hz}\right), 1.29$ (s, $6 \mathrm{H}) ;{ }^{13} \mathrm{C} \mathrm{NMR}\left(\mathrm{CDCl}_{3}, 75 \mathrm{MHz}\right): \delta 159.0,156.0,155.8,153.9,153.4,149.0,139.2,139.1$, $138.8,138.3,138.2,136.1,135.5,129.1,128.8,128.0,127.4,127.2,127.1,125.1,124.3$, $122.7,122.5,120.2,120.1,120.0,46.6,27.0 ;$ UV-Vis $\left(\mathrm{CH}_{2} \mathrm{Cl}_{2}\right) \lambda \mathrm{nm}\left(\varepsilon, \mathrm{M}^{-1} \mathrm{~cm}^{-1}\right)=318$ (33000), 308sh (26900), 293sh (19900), 279sh (17100); IR (KBr, cm-1) v = 3060 (w), 2958 (w), $2859(\mathrm{w}), 1565(\mathrm{~m}), 1470(\mathrm{~m}), 1422(\mathrm{~s}), 1207(\mathrm{w}), 906$ (s) $727(\mathrm{~s}) ; \mathrm{FAB}^{+} \mathrm{m} / z$ (nature of the peak, relative intensity) $425.1\left([\mathrm{M}+\mathrm{H}]^{+}, 100\right)$; Anal. Calcd for $\mathrm{C}_{31} \mathrm{H}_{24} \mathrm{~N}_{2}: \mathrm{C}, 87.70 ; \mathrm{H}$, 5.70; N, 6.60; Found: C, 87.52; H, 5.49; N, 6.44 .

4-(9,9-dimethylfluoren-2-yl)-6-phenyl-2,2'-bipyridine (4b). Minor isomer: $15 \mathrm{mg}$ (8\%) of $\mathbf{4 b}$ as a light yellow powder; ${ }^{1} \mathrm{H} \mathrm{NMR}\left(\mathrm{CDCl}_{3}, 300 \mathrm{MHz}\right): \delta 8.76-8.71(\mathrm{~m}, 3 \mathrm{H}), 8.26-$ $8.23(\mathrm{~m}, 2 \mathrm{H}), 8.05\left(\mathrm{~d}, 1 \mathrm{H},{ }^{4} \mathrm{~J}=1.3 \mathrm{~Hz}\right), 7.92-7.78(\mathrm{~m}, 5 \mathrm{H}), 7.59-7.45(\mathrm{~m}, 4 \mathrm{H}), 7.40-7.34$ (m, 3H), $1.59(\mathrm{~s}, 6 \mathrm{H}) ;{ }^{13} \mathrm{C} \mathrm{NMR}\left(\mathrm{CDCl}_{3}, 100 \mathrm{MHz}\right): \delta 157.3,156.6,156.4,154.6,154.2$, $150.8,149.2,140.4,139.7,138.6,137.9,137.1,129.2,128.9,127.9,127.3,126.6,123.9$, $122.8,121.8,121.6,120.6,120.5,118.8,117.6,47.3,27.4 ; \mathrm{UV}-\mathrm{Vis}\left(\mathrm{CH}_{2} \mathrm{Cl}_{2}\right) \lambda \mathrm{nm}\left(\varepsilon, \mathrm{M}^{-1} \mathrm{~cm}^{-}\right.$ $\left.{ }^{1}\right)=317$ (41000), 296 (34600), 268sh (26600); IR (KBr, cm-1) v= $3055(\mathrm{w}), 3034(\mathrm{w}), 2961$ (w), $2920(\mathrm{w}), 2864$ (w), 1584 (m), 1547 (m), 1468 (m), 1392 (m), 869 (m), 833 (m), 767 (s), 740 (s); $\mathrm{FAB}^{+} \mathrm{m} / \mathrm{z}$ (nature of the peak, relative intensity) $425.1\left([\mathrm{M}+\mathrm{H}]^{+}, 100\right)$; Anal. Calcd for $\mathrm{C}_{31} \mathrm{H}_{24} \mathrm{~N}_{2}$ : C, 87.70; H, 5.70; N, 6.60; Found: C, 87.51; H, 5.55; N, 6.41.

3-(N-methylcarbazol-3-yl)-6-phenyl-2,2'-bipyridine (5a). Prepared using procedure 1; from 5-phenyl-3-(2-pyridyl)-as-triazine (172 mg, $0.73 \mathrm{mmol})$, 3-ethynyl-N-methylcarbazole (150 mg, $0.73 \mathrm{mmol})$ and 1,2-dichlorobenzene $(1 \mathrm{ml})$; chromatography on silica gel eluting with dichloromethane to dichloromethane - methanol ( $/ / v 99 / 1)$ to give $130 \mathrm{mg}(43 \%)$ of $\mathbf{5 a}$ 
as light yellow powder after precipitation in pentane; ${ }^{1} \mathrm{H} \mathrm{NMR}\left(\mathrm{CDCl}_{3}, 300 \mathrm{MHz}\right): \delta 8.52$ (dt, $1 \mathrm{H},{ }^{3} J=4.9 \mathrm{~Hz},{ }^{4} J=1.3 \mathrm{~Hz}$ ), $8.18\left(\mathrm{~d}, 2 \mathrm{H},{ }^{3} J=7.0 \mathrm{~Hz}\right.$ ), $8.00-7.98$ (m, 2H), 7.93 (ABsys, $\left.2 \mathrm{H}, J_{\mathrm{AB}}=8.2 \mathrm{~Hz}, v_{0} \delta=33.0 \mathrm{~Hz}\right), 7.61-7.37(\mathrm{~m}, 7 \mathrm{H}), 7.25-7.21(\mathrm{~m}, 3 \mathrm{H}), 7.13(\mathrm{ddd}, 1 \mathrm{H}, J$ $=6.8 \mathrm{~Hz}, 3.8 \mathrm{~Hz}, 1.8 \mathrm{~Hz}), 3.81(\mathrm{~s}, 3 \mathrm{H}) ;{ }^{13} \mathrm{C} \mathrm{NMR}\left(\mathrm{CDCl}_{3}, 75 \mathrm{MHz}\right): \delta 159.3,156.0,155.4$, $149.1,141.4,140.2,139.8,139.3,136.0,135.9,130.3,128.9,128.8,127.5,127.2,125.9$, 125.1, 123.0, 122.9, 122.3, 121.2, 120.4, 119.9, 119.1, 108.7, 108.2, 29.2; UV-Vis $\left(\mathrm{CH}_{2} \mathrm{Cl}_{2}\right) \lambda$ $\mathrm{nm}\left(\varepsilon, \mathrm{M}^{-1} \mathrm{~cm}^{-1}\right)=352 \mathrm{sh}(8800), 319$ (20500), 300 (32500), 263sh (23800), 247sh (32700), 240sh (36900), 234 (37800); IR (KBr, cm-1) v = 3052 (w), $2935(\mathrm{w}), 1588(\mathrm{~m}), 1588(\mathrm{~m})$, $1549(\mathrm{~m}), 1477(\mathrm{~m}), 1450(\mathrm{~m}), 1418(\mathrm{~m}), 1254(\mathrm{~m}), 808(\mathrm{~m}), 744(\mathrm{~s}) ; \mathrm{FAB}^{+} \mathrm{m} / z$ (nature of the peak, relative intensity); $412.1\left([\mathrm{M}+\mathrm{H}]^{+}\right.$, 100); Anal. Calcd for $\mathrm{C}_{29} \mathrm{H}_{21} \mathrm{~N}_{3}: \mathrm{C}, 84.64 ; \mathrm{H}, 5.14$; N, 10.21; Found: C, 84.38; H, 4.92; N, 10.00 .

4-(N-methylcarbazol-3-yl)-6-phenyl-2,2'-bipyridine (5b). Minor isomer:35 mg (12\%) of 5b as a white solid; ${ }^{1} \mathrm{H}$ NMR $\left(\mathrm{CDCl}_{3}, 300 \mathrm{MHz}\right): \delta 8.79-8.73(\mathrm{~m}, 3 \mathrm{H}), 8.57\left(\mathrm{~d}, 1 \mathrm{H},{ }^{4} \mathrm{~J}=1.7\right.$ $\mathrm{Hz}), 8.27\left(\mathrm{~d}, 2 \mathrm{H},{ }^{3} J=7.5 \mathrm{~Hz}\right), 8.21\left(\mathrm{~d}, 1 \mathrm{H},{ }^{3} J=7.7 \mathrm{~Hz}\right), 8.12\left(\mathrm{~d}, 1 \mathrm{H},{ }^{4} J=1.3 \mathrm{~Hz}\right), 7.96(\mathrm{dd}$, $\left.1 \mathrm{H},{ }^{3} J=8.5 \mathrm{~Hz},{ }^{4} J=1.7 \mathrm{~Hz}\right), 7.88\left(\mathrm{td}, 1 \mathrm{H},{ }^{3} J=7.7 \mathrm{~Hz},{ }^{4} J=1.7 \mathrm{~Hz}\right), 7.59-7.28(\mathrm{~m}, 8 \mathrm{H})$, $3.86(\mathrm{~s}, 3 \mathrm{H}) ;{ }^{13} \mathrm{C} \mathrm{NMR}\left(\mathrm{CDCl}_{3}, 75 \mathrm{MHz}\right): \delta 157.1,156.7,156.1,151.1,149.0,141.6,141.5$, $139.8,136.9,129.5,129.0,128.7,127.2,126.2,125.1,123.7,123.4,122.9,121.6,120.6$, 119.4, 119.2, 118.5, 117.4, 108.9, 108.7, 29.2; UV-Vis $\left(\mathrm{CH}_{2} \mathrm{Cl}_{2}\right) \lambda \mathrm{nm}\left(\varepsilon, \mathrm{M}^{-1} \mathrm{~cm}^{-1}\right)=321$ (19000), 295 (41200), 261sh (27000), 248sh (36500), 240 (41600), 235sh (38500); IR (KBr, cm-1) $v=3052(\mathrm{w}), 2935(\mathrm{w}), 1595(\mathrm{~m}), 1567(\mathrm{~m}), 1487(\mathrm{~m}), 1402(\mathrm{~m}), 1247(\mathrm{~m}), 1126(\mathrm{~m})$, $920(\mathrm{~m}), 775(\mathrm{~m}), 725(\mathrm{~s}) ; \mathrm{FAB}^{+} \mathrm{m} / z$ (nature of the peak, relative intensity); $412.2\left([\mathrm{M}+\mathrm{H}]^{+}\right.$, 100); Anal. Calcd for $\mathrm{C}_{29} \mathrm{H}_{21} \mathrm{~N}_{3}$ : C, 84.64; H, 5.14; N, 10.21; Found: C, 84.57; H, 5.04; N, 10.15 . 
3-(pyren-1-yl)-6-phenyl-2,2'-bipyridine (6). Prepared using procedure 1; from 5-phenyl3-(2-pyridyl)-as-triazine (130 mg, $0.55 \mathrm{mmol}), 1$-ethynylpyrene $(120 \mathrm{mg}, 0.53 \mathrm{mmol})$ and 1,2-dichlorobenzene $(1 \mathrm{ml})$; chromatography on silica gel eluting with dichloromethane to dichloromethane - methanol ( $v / v 99 / 1)$ to give $45 \mathrm{mg}(20 \%)$ of 6 as a white powder after precipitation in pentane; ${ }^{1} \mathrm{H}$ NMR $\left(\mathrm{CDCl}_{3}, 300 \mathrm{MHz}\right): \delta 8.25\left(\mathrm{dt}, 2 \mathrm{H},{ }^{3} \mathrm{~J}=6.5 \mathrm{~Hz},{ }^{4} \mathrm{~J}=1.6\right.$ $\mathrm{Hz}), 8.22-8.10(\mathrm{~m}, 4 \mathrm{H}), 8.06\left(\mathrm{~d}, 2 \mathrm{H},{ }^{4} J=1.3 \mathrm{~Hz}\right), 7.96\left(\mathrm{~d}, 2 \mathrm{H},{ }^{4} J=1.1 \mathrm{~Hz}\right), 7.93$ (ABsys, $\left.2 \mathrm{H}, J_{\mathrm{AB}}=7.7 \mathrm{~Hz}, v_{0} \delta=43.6 \mathrm{~Hz}\right), 7.92(\mathrm{~s}, 2 \mathrm{H}), 7.58-7.45(\mathrm{~m}, 4 \mathrm{H}), 7.34\left(\mathrm{td}, 1 \mathrm{H},{ }^{3} J=7.7 \mathrm{~Hz}\right.$, $\left.{ }^{4} J=1.8 \mathrm{~Hz}\right), 6.88(\mathrm{ddd}, 1 \mathrm{H}, J=7.5 \mathrm{~Hz}, 4.8 \mathrm{~Hz}, 1.2 \mathrm{~Hz}) ;{ }^{13} \mathrm{C} \mathrm{NMR}\left(\mathrm{CDCl}_{3}, 75 \mathrm{MHz}\right): \delta 158.2$, $156.9,156.5,149.0,141.3,139.2,135.7,135.3,134.1,131.5,131.0,130.7,129.3,128.9$, $128.8,128.3,127.7,127.6,127.5,127.4,126.1,125.3,125.1,125.0,124.9,124.8,124.6$, 124.5, 122.3, 119.6; UV-Vis $\left(\mathrm{CH}_{2} \mathrm{Cl}_{2}\right) \lambda \mathrm{nm}\left(\varepsilon, \mathrm{M}^{-1} \mathrm{~cm}^{-1}\right)=349$ (40100), 340sh (31300), 322sh (18600), 280 (51600), 273sh (41600), 244 (70000); IR (KBr, cm-1) v = 3049 (w), 1582 (m), $1550(\mathrm{~m}), 1472(\mathrm{~m}), 1447(\mathrm{~m}), 1419(\mathrm{~m}), 1141(\mathrm{~m}), 846(\mathrm{~s}), 749(\mathrm{~s}) ; \mathrm{FAB}^{+} \mathrm{m} / z$ (nature of the peak, relative intensity) $433.1\left([\mathrm{M}+\mathrm{H}]^{+}, 100\right)$; Anal. Calcd for $\mathrm{C}_{32} \mathrm{H}_{20} \mathrm{~N}_{2}: \mathrm{C}, 88.86 ; \mathrm{H}$, 4.66; N, 6.48; Found: C, 88.24; H, 4.80; N, 6.17.

3-(N,N-dibutyl-4-aminophenyl)-6-phenyl-2,2'-bipyridine (8a). Prepared using procedure 1; from 5-phenyl-3-(2-pyridyl)-as-triazine $\quad(190 \quad \mathrm{mg}, \quad 0.81 \quad \mathrm{mmol}), \quad \mathrm{N}, \mathrm{N}-$ dibutyl-4ethynylbenzenamine $(165 \mathrm{mg}, 0.72 \mathrm{mmol})$ and 1,2-dichlorobenzene $(0.5 \mathrm{ml})$; chromatography on silica gel eluting with dichloromethane - petroleum ether ( $v / v 50 / 50$ to $1 / 0)$ to give $270 \mathrm{mg}$ (86 \%) of 8a as a yellow oil; ${ }^{1} \mathrm{H}$ NMR $\left(\mathrm{CDCl}_{3}, 300 \mathrm{MHz}\right): \delta 8.61$ (ddd, $1 \mathrm{H}, J=4.9 \mathrm{~Hz}, 1.6$ $\mathrm{Hz}, 0.9 \mathrm{~Hz}), 8.11\left(\mathrm{~d}, 2 \mathrm{H},{ }^{3} J=6.8 \mathrm{~Hz}\right), 7.82\left(\right.$ ABsys, $\left.2 \mathrm{H}, J_{\mathrm{AB}}=8.2 \mathrm{~Hz}, v_{0} \delta=12.5 \mathrm{~Hz}\right), 7.64$ $\left(\mathrm{td}, 1 \mathrm{H},{ }^{3} J=7.7 \mathrm{~Hz},{ }^{4} J=1.7 \mathrm{~Hz}\right), 7.53-7.36(\mathrm{~m}, 4 \mathrm{H}), 7.20(\mathrm{ddd}, 1 \mathrm{H}, J=7.2 \mathrm{~Hz}, 4.9 \mathrm{~Hz}, 1.2$ $\mathrm{Hz}), 7.01\left(\mathrm{~d}, 2 \mathrm{H},{ }^{3} \mathrm{~J}=8.9 \mathrm{~Hz}\right), 6.52\left(\mathrm{~d}, 2 \mathrm{H},{ }^{3} \mathrm{~J}=8.7 \mathrm{~Hz}\right), 3.24\left(\mathrm{t}, 4 \mathrm{H},{ }^{3} J=7.5 \mathrm{~Hz}\right), 1.61-1.51$ $(\mathrm{m}, 4 \mathrm{H}), 1.40-1.28(\mathrm{~m}, 4 \mathrm{H}), 0.95\left(\mathrm{t}, 6 \mathrm{H},{ }^{3} \mathrm{~J}=7.1 \mathrm{~Hz}\right) ;{ }^{13} \mathrm{C} \mathrm{NMR}\left(\mathrm{CDCl}_{3}, 75 \mathrm{MHz}\right): \delta$ 159.6, 
$155.5,154.8,149.2,147.4,139.4,139.0,135.9,135.3,130.6,128.7,127.1,125.6,125.1$, $122.3,119.9,111.5,50.8,29.5,20.5,14.1 ;$ UV-Vis $\left(\mathrm{CH}_{2} \mathrm{Cl}_{2}\right) \lambda \mathrm{nm}\left(\varepsilon, \mathrm{M}^{-1} \mathrm{~cm}^{-1}\right)=350$ (21900), 262 (22700); IR (KBr, cm-1) v = 3060 (w), 2955 (s), 2870 (m), 1608 (s), 1522 (s), $1454(\mathrm{~m}), 1367(\mathrm{~m}), 1197(\mathrm{~m}), 803(\mathrm{~s}), 754(\mathrm{~s}) ; \mathrm{FAB}^{+} \mathrm{m} / \mathrm{z}$ (nature of the peak, relative intensity) $436.2\left([\mathrm{M}+\mathrm{H}]^{+}, 100\right), 378\left(\left[\mathrm{M}-\mathrm{C}_{4} \mathrm{H}_{9}\right]^{+}, 30\right)$; Anal. Calcd for $\mathrm{C}_{30} \mathrm{H}_{33} \mathrm{~N}_{3}: \mathrm{C}, 82.72 ; \mathrm{H}$, 7.64; N, 9.65; Found: C, 82.44; H, 7.37; N, 9.41.

4-(N,N-dibutyl-4-aminophenyl)-6-phenyl-2,2'-bipyridine (8b). Minor isomer: $15 \mathrm{mg}$ (5 \%) of $\mathbf{8 b}$ as a pale yellow solid; ${ }^{1} \mathrm{H}$ NMR $\left(\mathrm{CDCl}_{3}, 400 \mathrm{MHz}\right): \delta 8.73-8.71(\mathrm{~m}, 1 \mathrm{H}), 8.67(\mathrm{~d}$, $\left.1 \mathrm{H},{ }^{3} J=7.5 \mathrm{~Hz}\right), 8.62\left(\mathrm{~d}, 1 \mathrm{H},{ }^{4} J=1.7 \mathrm{~Hz}\right), 8.21-8.19(\mathrm{~m}, 2 \mathrm{H}), 7.96\left(\mathrm{~d}, 1 \mathrm{H},{ }^{4} J=1.7 \mathrm{~Hz}\right)$, $7.85\left(\mathrm{td}, 1 \mathrm{H},{ }^{3} J=7.8 \mathrm{~Hz},{ }^{4} J=1.8 \mathrm{~Hz}\right), 7.76\left(\mathrm{~d}, 2 \mathrm{H},{ }^{3} J=9.1 \mathrm{~Hz}\right), 7.54-7.43(\mathrm{~m}, 3 \mathrm{H}), 7.32$ (ddd, $1 \mathrm{H}, J=7.5 \mathrm{~Hz}, 5.0 \mathrm{~Hz}, 1.3 \mathrm{~Hz}), 6.75\left(\mathrm{~d}, 2 \mathrm{H},{ }^{3} J=8.7 \mathrm{~Hz}\right), 3.35$ (t, $\left.4 \mathrm{H},{ }^{3} J=7.7 \mathrm{~Hz}\right), 1.66$ $-1.59(\mathrm{~m}, 4 \mathrm{H}), 1.44-1.35(\mathrm{~m}, 4 \mathrm{H}), 0.99\left(\mathrm{t}, 6 \mathrm{H},{ }^{3} J=7.5 \mathrm{~Hz}\right) ;{ }^{13} \mathrm{C} \mathrm{NMR}\left(\mathrm{CDCl}_{3}, 75 \mathrm{MHz}\right): \delta$ $157.0,156.9,156.1,150.1,149.1,149.0,140.2,136.9,128.9,128.8,128.2,127.2,124.6$, 123.7, 121.6, 117.2, 116.2, 111.8, 50.9, 29.6, 20.5, 14.2; UV-Vis $\left(\mathrm{CH}_{2} \mathrm{Cl}_{2}\right) \lambda \mathrm{nm}\left(\varepsilon, \mathrm{M}^{-1} \mathrm{~cm}^{-1}\right)$ $=356$ (28200), 292 (22400), 264 (24600), 246sh (25800); IR (KBr, cm-1) v = 3054 (w), 2955 (m), 2925 (m), 2856 (m), 1580 (s), 1524 (s), 1362 (m), 1202 (m), 1079 (m), 813 (s), 774 (s); $\mathrm{FAB}^{+} \mathrm{m} / \mathrm{z}$ (nature of the peak, relative intensity) $436.2\left([\mathrm{M}+\mathrm{H}]^{+}, 100\right)$; Anal. Calcd for $\mathrm{C}_{30} \mathrm{H}_{33} \mathrm{~N}_{3}$ : C, 82.72; H, 7.64; N, 9.65; Found: C, 82.69; H, 7.53; N, 9.52.

4-(2,2':6',2"-terpyridine)-6-phenyl-2,2'-bipyridine (9). Prepared using procedure 1; from 5-phenyl-3-(2-pyridyl)-as-triazine (150 mg, $0.64 \mathrm{mmol}), 4^{\prime}$-ethynyl-2,2':6',2"-terpyridine (165 $\mathrm{mg}, 0.64 \mathrm{mmol})$ and 1,2-dichlorobenzene (1 ml); chromatography on aluminium oxide eluting with dichloromethane - petroleum ether $(v / v 50 / 50)$ to give $25 \mathrm{mg}(8 \%)$ of 9 as white powder; ${ }^{1} \mathrm{H}$ NMR $\left(\mathrm{CDCl}_{3}, 400 \mathrm{MHz}\right): \delta 8.92(\mathrm{~s}, 2 \mathrm{H}), 8.88\left(\mathrm{~d}, 1 \mathrm{H},{ }^{4} \mathrm{~J}=1.0 \mathrm{~Hz}\right), 8.77-8.70$ 
$(\mathrm{m}, 6 \mathrm{H}), 8.28\left(\mathrm{~d}, 2 \mathrm{H},{ }^{3} J=7.0 \mathrm{~Hz}\right), 8.22\left(\mathrm{~d}, 1 \mathrm{H},{ }^{4} J=1.0 \mathrm{~Hz}\right), 7.90(\mathrm{~m}, 3 \mathrm{H}), 7.55(\mathrm{~m}, 2 \mathrm{H}), 7.48$ (m, 1H), $7.38(\mathrm{~m}, 3 \mathrm{H}) ;{ }^{13} \mathrm{C} \mathrm{NMR}\left(\mathrm{CDCl}_{3}, 100 \mathrm{MHz}\right): \delta 157.7,156.8,156.4,156.2,156.0$, $149.3,149.2,148.7,148.4,139.3,137.2,137.1,129.4,128.9,127.4,124.2,124.1,121.8$, 121.6, 119.4, 118.8, 117.9; UV-Vis $\left(\mathrm{CH}_{2} \mathrm{Cl}_{2}\right) \lambda \mathrm{nm}\left(\varepsilon, \mathrm{M}^{-1} \mathrm{~cm}^{-1}\right)=315$ (15000), 293sh (29000), 277 (36000), 252sh (47800), 243 (53500); IR (KBr, cm-1) v = 3052 (w), 1581 (s), $1469(\mathrm{~m}), 1382(\mathrm{~m}), 1268$ (w), $870(\mathrm{~m}), 788$ (s), 732 (s); $\mathrm{FAB}^{+} \mathrm{m} / z$ (nature of the peak, relative intensity) $464.2\left([\mathrm{M}+\mathrm{H}]^{+}, 100\right)$; Anal. Calcd for $\mathrm{C}_{31} \mathrm{H}_{21} \mathrm{~N}_{5}: \mathrm{C}, 80.32 ; \mathrm{H}, 4.57 ; \mathrm{N}$, 15.11; Found: C, 80.09; H, 4.36; N, 14.84.

12a. Prepared using procedure 1; from 5-phenyl-3-(2-pyridyl)-as-triazine (325 mg, 1.39 mmol), 1,4-diethynyl-2,5-didodecyloxybenzene $\quad\left(\begin{array}{llll}150 & \mathrm{mg}, & 0.55 & \mathrm{mmol}\end{array}\right)$ and 1,2dichlorobenzene $(1 \mathrm{ml})$; chromatography on aluminium oxide eluting with dichloromethane petroleum ether $(v / v 25 / 75$ to $1 / 0)$ to give $80 \mathrm{mg}(21 \%)$ of $\mathbf{1 2 a}$ as a white powder after reprecipitation in dichloromethane - methanol; ${ }^{1} \mathrm{H} \mathrm{NMR}\left(\mathrm{CDCl}_{3}, 300 \mathrm{MHz}\right): \delta 8.51$ (dt, $2 \mathrm{H}$, $\left.{ }^{3} J=4.7 \mathrm{~Hz},{ }^{4} J=1.3 \mathrm{~Hz}\right), 8.17-8.14(\mathrm{~m}, 4 \mathrm{H}), 7.85$ (ABsys, $4 \mathrm{H}, J_{\mathrm{AB}}=8.1 \mathrm{~Hz}, \mathrm{v}_{0} \delta=16.4$ Hz), $7.69-7.62(\mathrm{~m}, 4 \mathrm{H}), 7.52-7.39(\mathrm{~m}, 6 \mathrm{H}), 7.20-7.15(\mathrm{~m}, 2 \mathrm{H}), 6.62(\mathrm{~s}, 2 \mathrm{H}), 3.50(\mathrm{t}, 4 \mathrm{H}$, $\left.{ }^{3} J=6.8 \mathrm{~Hz}\right), 1.43-1.34(\mathrm{~m}, 4 \mathrm{H}), 1.25-1.12(\mathrm{~m}, 4 \mathrm{H}), 0.81\left(\mathrm{t}, 6 \mathrm{H},{ }^{3} J=7.6 \mathrm{~Hz}\right) ;{ }^{13} \mathrm{C} \mathrm{NMR}$ $\left(\mathrm{CDCl}_{3}, 75 \mathrm{MHz}\right): \delta 159.2,156.2,155.6,149.7,148.6,140.5,139.3,135.7,131.7,129.0$, $128.9,128.8,127.2,124.3,122.3,119.3,115.8,68.6,31.1,19.1,13.8 ; \mathrm{UV}-\mathrm{V}$ is $\left(\mathrm{CH}_{2} \mathrm{Cl}_{2}\right) \lambda \mathrm{nm}$ $\left(\varepsilon, \mathrm{M}^{-1} \mathrm{~cm}^{-1}\right)=324 \mathrm{sh}(19400), 275$ (38500), 233sh (38000); IR (KBr, cm-1) $v=3058(\mathrm{w})$ $2963(\mathrm{w}), 2933$ (w), 2863 (w), 1587 (m), 1564 (m), 1457 (m), 1377 (m), 1213 (s), 1044 (m), $753(\mathrm{~s}) ; \mathrm{FAB}^{+} \mathrm{m} / \mathrm{z}$ (nature of the peak, relative intensity) $683.3\left([\mathrm{M}+\mathrm{H}]^{+}, 100\right), 625.2$ ([M$\left.\mathrm{C}_{4} \mathrm{H}_{9}\right]^{+}, 30$ ); Anal. Calcd for $\mathrm{C}_{46} \mathrm{H}_{42} \mathrm{~N}_{4} \mathrm{O}_{2}$ : C, 80.91; H, 6.20; N, 8.20; Found: C, 80.75; H, $5.98 ; \mathrm{N}, 7.93$. 
12b. Minor isomer: $40 \mathrm{mg}(11 \%)$ of $\mathbf{1 2 b}$ as a white powder after reprecipitation in dichloromethane - methanol; ${ }^{1} \mathrm{H}$ NMR $\left(\mathrm{CDCl}_{3}, 300 \mathrm{MHz}\right): \delta 8.72-8.69(\mathrm{~m}, 2 \mathrm{H}), 8.60(\mathrm{~d}$, $\left.1 \mathrm{H},{ }^{4} J=1.5 \mathrm{~Hz}\right), 8.53(\mathrm{ddd}, 1 \mathrm{H}, J=4.8 \mathrm{~Hz}, 1.7 \mathrm{~Hz}, 1.0 \mathrm{~Hz}), 8.23-8.17$ (m, 4H), 8.09 (d, 1H, ${ }^{4} J=1.5 \mathrm{~Hz}$ ), 7.89 (ABsys, 2H, $\left.J_{\mathrm{AB}}=8.19 \mathrm{~Hz}, v_{0} \delta=19.3 \mathrm{~Hz}\right), 7.90-7.84(\mathrm{~m}, 1 \mathrm{H}), 7.77-$ $7.66(\mathrm{~m}, 2 \mathrm{H}), 7.55-7.41(\mathrm{~m}, 6 \mathrm{H}), 7.35-7.31(\mathrm{~m}, 1 \mathrm{H}), 7.19$ (ddd, 1H, J=7.3 Hz, 4.9 Hz, 1.5 $\mathrm{Hz}), 7.01(\mathrm{~s}, 1 \mathrm{H}), 6.86(\mathrm{~s}, 1 \mathrm{H}) 3.81\left(\mathrm{t}, 2 \mathrm{H},{ }^{3} \mathrm{~J}=6.3 \mathrm{~Hz}\right), 3.74\left(\mathrm{t}, 2 \mathrm{H},{ }^{3} J=6.6 \mathrm{~Hz}\right), 1.67-1.20$ $(\mathrm{m}, 8 \mathrm{H}), 0.87-0.80(\mathrm{~m}, 6 \mathrm{H}) ;{ }^{13} \mathrm{C} \mathrm{NMR}\left(\mathrm{CDCl}_{3}, 75 \mathrm{MHz}\right): \delta 159.1,156.8,156.3,156.2$, $155.8,155.7,150.3,150.2,149.1,148.8,148.1,140.5,139.9,139.3,136.9,136.0,131.5$, $130.7,129.1,129.0,128.8,128.2,127.3,127.1,124.3,123.7,122.6,121.6,119.9,119.3$, $116.8,114.7,69.2,69.1,31.5,31.4,19.4,19.2,13.9,13.8 ; \mathrm{UV}-\mathrm{Vis}\left(\mathrm{CH}_{2} \mathrm{Cl}_{2}\right) \lambda \mathrm{nm}\left(\varepsilon, \mathrm{M}^{-1} \mathrm{~cm}^{-}\right.$ $\left.{ }^{1}\right)=335 \mathrm{sh}$ (17000), 278 (39800), 264 (41000), 237 (47000); IR (KBr, cm-1) v = $3058(\mathrm{w})$, $2958(\mathrm{~m}), 2870(\mathrm{~m}), 1583(\mathrm{~m}), 1509$ (m), $1456(\mathrm{~m}), 1380(\mathrm{~m}), 1207$ (s), $1024(\mathrm{~m}), 758(\mathrm{~s})$, 689 (m); EIMS $m / z$ (nature of the peak, relative intensity) $682.2\left([\mathrm{M}]^{+}, 100\right)$; Anal. Calcd for $\mathrm{C}_{46} \mathrm{H}_{42} \mathrm{~N}_{4} \mathrm{O}_{2}:$ C, 80.91; H, 6.20; N, 8.20; Found: C, 80.75; H, 5.93; N, 8.02.

3,4-bis-(N,N-dibutyl-4-aminophenyl)-6-phenyl-2,2'-bipyridine (13). Prepared using procedure 1; from 5-phenyl-3-(2-pyridyl)-as-triazine (125 mg, $0.53 \mathrm{mmol}), \mathrm{N}, \mathrm{N}$-dibutyl-4-(2(4-(dibutylamino)phenyl)ethynyl)benzenamine (230 $\mathrm{mg}, 0.53 \mathrm{mmol})$ and 1,2-dichlorobenzene (1 ml); chromatography on aluminium oxide eluting with dichloromethane - petroleum ether $(v / v 0 / 1$ to $50 / 50)$ to afford $194 \mathrm{mg}(57 \%)$ of 13 as a white to light pink powder; ${ }^{1} \mathrm{H}$ NMR $\left(\mathrm{CDCl}_{3}, 400 \mathrm{MHz}\right): \delta 8.59\left(\mathrm{~d}, 1 \mathrm{H},{ }^{3} J=4.5 \mathrm{~Hz}\right), 8.11\left(\mathrm{~d}, 2 \mathrm{H},{ }^{3} J=7.0 \mathrm{~Hz}\right), 7.80(\mathrm{~s}, 1 \mathrm{H}), 7.49-$ $7.35(\mathrm{~m}, 4 \mathrm{H}), 7.19\left(\mathrm{~d}, 1 \mathrm{H},{ }^{3} J=8.0 \mathrm{~Hz}\right), 7.09$ (ddd, $\left.1 \mathrm{H}, J=7.5 \mathrm{~Hz}, 4.8 \mathrm{~Hz}, 1.3 \mathrm{~Hz}\right), 6.78$ (ABsys, $\left.4 \mathrm{H}, J_{\mathrm{AB}}=9.0 \mathrm{~Hz}, v_{0} \delta=220.6 \mathrm{~Hz}\right), 6.55\left(\right.$ ABsys, $4 \mathrm{H}, J_{\mathrm{AB}}=8.8 \mathrm{~Hz}, v_{0} \delta=144.5 \mathrm{~Hz}$ ), $3.24\left(\mathrm{t}, 4 \mathrm{H},{ }^{3} J=7.5 \mathrm{~Hz}\right), 3.17\left(\mathrm{t}, 4 \mathrm{H},{ }^{3} J=7.5 \mathrm{~Hz}\right), 1.59-1.44(\mathrm{~m}, 8 \mathrm{H}), 1.38-1.24(\mathrm{~m}, 8 \mathrm{H})$, $0.95\left(\mathrm{t}, 6 \mathrm{H},{ }^{3} \mathrm{~J}=7.3 \mathrm{~Hz}\right), 0.92\left(\mathrm{t}, 6 \mathrm{H},{ }^{3} J=7.5 \mathrm{~Hz}\right) ;{ }^{13} \mathrm{C} \mathrm{NMR}\left(\mathrm{CDCl}_{3}, 100 \mathrm{MHz}\right): \delta 160.2$, 
$157.7,155.3,150.5,149.0,147.5,146.9,139.9,135.3,133.3,132.2,130.7,128.6,128.5$, $127.3,126.3,125.2,124.7,121.6,121.5,111.7,111.1,50.8,50.7,29.5,29.4,20.5,20.4,14.2$, 14.1; UV-Vis $\left(\mathrm{CH}_{2} \mathrm{Cl}_{2}\right) \lambda \mathrm{nm}\left(\varepsilon, \mathrm{M}^{-1} \mathrm{~cm}^{-1}\right)=355 \mathrm{sh}$ (19200), 331 (2860), 263 (30600); IR $(\mathrm{KBr}, \mathrm{cm}-1) v=3039(\mathrm{w}), 2955(\mathrm{~m}), 2929(\mathrm{~m}), 2870(\mathrm{~m}), 1605$ (s), 1517 (s), 1463 (m), 1364 (s), $1285(\mathrm{~m}), 1198(\mathrm{~s}), 1095(\mathrm{~m}), 818(\mathrm{~s}) ; \mathrm{FAB}^{+} \mathrm{m} / \mathrm{z}$ (nature of the peak, relative intensity) $639.3\left([\mathrm{M}+\mathrm{H}]^{+}, 100\right)$; Anal. Calcd for $\mathrm{C}_{44} \mathrm{H}_{54} \mathrm{~N}_{4}: \mathrm{C}, 82.71 ; \mathrm{H}, 8.52 ; \mathrm{N}, 8.77$; Found: C, 82.54; H, 8.29; N, 8.47.

3-phenyl-1-(pyridin-2-yl)isoquinoline (14). A Schlenk tube was charged with 5-phenyl-3(2-pyridyl)-as-triazine (500 $\mathrm{mg}, 2.13 \mathrm{mmol})$, o-dichlorobenzene $(4 \mathrm{ml})$, argon flushed and heated up to $160{ }^{\circ} \mathrm{C}$. Anthranilic acid $(880 \mathrm{mg}, 6.42 \mathrm{mmol})$ suspended in $6 \mathrm{ml} \mathrm{o}$ dichlorobenzene as well as isoamyl nitrite $(900 \mathrm{ul}, 6.70 \mathrm{mmol})$ in $4 \mathrm{ml} o$-dichlorobenzene were added dropwise via 2 syringes within $15 \mathrm{~min}$. The mixture was stirred at this temperature for 4 hours. The solvent was then removed via high vacuum, the residue treated with water and extracted with dichloromethane. After evaporation the dark residue was purified by column chromatography on silica gel eluting with dichloromethane - methanol $(v / v 99.5 / 0.5)$ to give $120 \mathrm{mg}(20 \%)$ of 14 as a pale yellow solid after reprecipitation from diethyl ether - pentane; ${ }^{1} \mathrm{H}$ NMR $\left(\mathrm{CDCl}_{3}, 300 \mathrm{MHz}\right): \delta 8.73\left(\mathrm{~d}, 1 \mathrm{H},{ }^{3} J=4.3 \mathrm{~Hz}\right), 8.24(\mathrm{~d}, 1 \mathrm{H}$, $\left.{ }^{3} J=7.9 \mathrm{~Hz}\right), 7.81\left(\mathrm{td}, 1 \mathrm{H},{ }^{3} J=7.7 \mathrm{~Hz},{ }^{4} J=1.7 \mathrm{~Hz}\right), 7.57-7.54(\mathrm{~m}, 2 \mathrm{H}), 7.42-7.31(\mathrm{~m}, 9 \mathrm{H})$; ${ }^{13} \mathrm{C} \mathrm{NMR}\left(\mathrm{CDCl}_{3}, 75 \mathrm{MHz}\right): \delta 161.5,155.2,150.0,149.7,138.2,136.7,130.1,129.3,129.1$, 129.0, 128.5, 127.7, 125.6, 123.9, 121.9; UV-Vis $\left(\mathrm{CH}_{2} \mathrm{Cl}_{2}\right) \lambda \mathrm{nm}\left(\varepsilon, \mathrm{M}^{-1} \mathrm{~cm}^{-1}\right)=312(7400)$, 242 (16500); IR (KBr, cm-1) v = 3066 (w), 2089 (w), $2026(\mathrm{w}), 1963$ (w), 1605 (m), 1497 (m), $1443(\mathrm{~m}), 1349(\mathrm{~m}), 1294(\mathrm{~m}), 997(\mathrm{~m}), 814(\mathrm{~m}), 756(\mathrm{~m}), 692(\mathrm{~m}) \mathrm{FAB}^{+} \mathrm{m} / z$ (nature of the peak, relative intensity) $282.1\left([\mathrm{M}+\mathrm{H}]^{+}, 100\right)$, Anal. Calcd for $\mathrm{C}_{20} \mathrm{H}_{14} \mathrm{~N}_{2}: \mathrm{C}, 85.08 ; \mathrm{H}$, 5.00; N, 9.92; Found: C, 84.95; H, 4.92; N, 9.75. 
4-(tributylstannyl)-6-phenyl-2,2'-bipyridine (15a). Prepared using procedure 1; from 5phenyl-3-(2-pyridyl)-as-triazine (384 mg, $1.64 \mathrm{mmol})$, tributyl(ethynyl)stannane (1,00 g, 3.28 mmol) and 1,2-dichlorobenzene $(1.5 \mathrm{ml})$; chromatography on silica gel eluting with dichloromethane - petroleum ether $(v / \mathrm{v} 0 / 1$ to $1 / 0)$ to give $650 \mathrm{mg}(76 \%)$ of $\mathbf{1 5 a}$ as a pale yellow oil; ${ }^{1} \mathrm{H}$ NMR $\left(\mathrm{CDCl}_{3}, 300 \mathrm{MHz}\right): \delta 8.70(\mathrm{ddd}, 1 \mathrm{H}, J=4.8 \mathrm{~Hz}, 1.8 \mathrm{~Hz}, 0.9 \mathrm{~Hz}), 8.62$ (dt, $\left.1 \mathrm{H},{ }^{3} J=8.0 \mathrm{~Hz},{ }^{4} J=1.0 \mathrm{~Hz}\right), 8.47\left(\mathrm{~d}\right.$ with ${ }^{119} \mathrm{Sn}$ satellites, $1 \mathrm{H}, J_{\mathrm{Sn}-\mathrm{H}}=18.6 \mathrm{~Hz},{ }^{4} J=0.4$ $\mathrm{Hz}), 8.15-8.11(\mathrm{~m}, 2 \mathrm{H}), 7.85\left(\mathrm{~d}\right.$ with ${ }^{119} \mathrm{Sn}$ satellites, $\left.1 \mathrm{H}, J_{\mathrm{Sn}-\mathrm{H}}=18.6 \mathrm{~Hz},{ }^{4} J=0.4 \mathrm{~Hz}\right), 7.83$ $\left(\operatorname{td}, 1 \mathrm{H},{ }^{3} J=7.8 \mathrm{~Hz},{ }^{4} J=1.9 \mathrm{~Hz}\right), 7.54-7.40(\mathrm{~m}, 3 \mathrm{H}), 7.30(\mathrm{ddd}, 1 \mathrm{H}, J=7.4 \mathrm{~Hz}, 4.8 \mathrm{~Hz}, 1.2$ $\mathrm{Hz}), 1.65-1.54(\mathrm{~m}, 6 \mathrm{H}), 1.43-1.30(\mathrm{~m}, 6 \mathrm{H}), 1.20-1.16(\mathrm{~m}, 6 \mathrm{H}), 0.90\left(\mathrm{t}, 9 \mathrm{H},{ }^{3} J=7.4 \mathrm{~Hz}\right)$; ${ }^{13} \mathrm{C}$ NMR $\left(\mathrm{CDCl}_{3}, 75 \mathrm{MHz}\right): \delta 157.2,154.9,154.8,153.8,149.2,140.1,136.9,128.9,128.8$, $128.7,127.3,127.2,123.6,121.7,29.2,27.5,13.8,9.9$.

4-iodo-6-phenyl-2,2'-bipyridine (15b). To a solution of 4-(tributylstannyl)-6-phenyl-2,2'bipyridine $(650 \mathrm{mg}, 1.25 \mathrm{mmol})$ in chloroform $(30 \mathrm{ml})$ was added dropwise a solution of iodine $(320 \mathrm{mg}, 1.25 \mathrm{mmol})$ in chloroform $(15 \mathrm{ml})$. The reaction mixture was stirred at room temperature for $2 \mathrm{~h}$ and then treated twice with saturated aqueous $\mathrm{Na}_{2} \mathrm{SO}_{3}$. The organic layer was dried over anhydrous magnesium sulphate, evaporated and the residue was purified by chromatography on silica gel eluting with dichloromethane - petroleum ether $(v / v$ v $0 / 1$ to $1 / 0)$ and the residue was crystallised in pentane to afford $400 \mathrm{mg}(89 \%)$ of $\mathbf{1 5 b}$ as white crystals; ${ }^{1} \mathrm{H}$ NMR $\left(\mathrm{CDCl}_{3}, 300 \mathrm{MHz}\right): \delta 8.78\left(\mathrm{~d}, 1 \mathrm{H},{ }^{4} J=1.5 \mathrm{~Hz}\right), 8.69(\mathrm{ddd}, 1 \mathrm{H}, J=4.8 \mathrm{~Hz}, 1.7 \mathrm{~Hz}$, $0.9 \mathrm{~Hz}), 8.59\left(\mathrm{dt}, 1 \mathrm{H},{ }^{3} J=8.0 \mathrm{~Hz},{ }^{4} J=1.0 \mathrm{~Hz}\right), 8.14\left(\mathrm{~d}, 1 \mathrm{H},{ }^{4} J=1.3 \mathrm{~Hz}\right), 8.12-8.08(\mathrm{~m}, 2 \mathrm{H})$, $7.85\left(\mathrm{td}, 1 \mathrm{H},{ }^{3} J=7.9 \mathrm{~Hz},{ }^{4} J=1.7 \mathrm{~Hz}\right), 7.54-7.43(\mathrm{~m}, 3 \mathrm{H}), 7.34(\mathrm{ddd}, 1 \mathrm{H}, J=7.5 \mathrm{~Hz}, 4.8 \mathrm{~Hz}$, $1.2 \mathrm{~Hz}) ;{ }^{13} \mathrm{C} \mathrm{NMR}\left(\mathrm{CDCl}_{3}, 75 \mathrm{MHz}\right): \delta 157.4,156.3,155.1,149.3,138.1,137.1,129.7,129.6$, $129.0,128.6,127.2,124.4,121.7,107.4 ; \mathrm{FAB}^{+} \mathrm{m} / \mathrm{z}$ (nature of the peak, relative intensity) 
359.1 $\left([\mathrm{M}+\mathrm{H}]^{+}, 100\right), 231.1\left([\mathrm{M}-\mathrm{I}]^{+}, 15\right)$; Anal. Calcd for $\mathrm{C}_{16} \mathrm{H}_{11} \mathrm{IN}_{2}: \mathrm{C}, 53.65 ; \mathrm{H}, 3.10 ; \mathrm{N}$, 7.82; Found: C, 53.37; H, 2.72; N, 7.54.

\section{4-(2-(N,N-dibutyl-4-aminophenyl)ethynyl)-6-phenyl-2,2'-bipyridine (21). Prepared} using procedure 2; from 4-iodo-6-phenyl-2,2'-bipyridine (100 mg, $0.28 \mathrm{mmol}$ ), N,N-dibutyl4-ethynylbenzenamine (71 mg, $0.31 \mathrm{mmol}), \mathrm{Pd}\left(\mathrm{PPh}_{3}\right)_{2} \mathrm{Cl}_{2}(12 \mathrm{mg}, 0.017 \mathrm{mmol}), \mathrm{CuI}(3 \mathrm{mg}$, $0.016 \mathrm{mmol})$, THF $(10 \mathrm{ml})$ and ${ }^{i} \mathrm{Pr}_{2} \mathrm{NH}(2 \mathrm{ml})$; chromatography on aluminium oxide eluting with dichloromethane to give $105 \mathrm{mg}(82 \%)$ of 21 as a yellow oil; ${ }^{1} \mathrm{H}$ NMR $\left(\mathrm{CDCl}_{3}, 300\right.$ MHz): $\delta 8.72(\mathrm{ddd}, 1 \mathrm{H}, J=4.8 \mathrm{~Hz}, 1.7 \mathrm{~Hz}, 0.9 \mathrm{~Hz}), 8.64\left(\mathrm{~d}, 1 \mathrm{H},{ }^{3} J=7.9 \mathrm{~Hz}\right), 8.46\left(\mathrm{~d}, 1 \mathrm{H},{ }^{4} J\right.$ $=1.1 \mathrm{~Hz}), 8.19-8.15(\mathrm{~m}, 2 \mathrm{H}), 7.85\left(\mathrm{dt}, 1 \mathrm{H},{ }^{3} J=7.7 \mathrm{~Hz},{ }^{4} J=1.9 \mathrm{~Hz}\right), 7.83\left(\mathrm{~d}, 1 \mathrm{H},{ }^{4} J=1.3\right.$ Hz), $7.55-7.41(\mathrm{~m}, 5 \mathrm{H}), 7.33(\mathrm{ddd}, 1 \mathrm{H}, J=7.5 \mathrm{~Hz}, 4.8 \mathrm{~Hz}, 1.2 \mathrm{~Hz}), 6.61\left(\mathrm{~d}, 2 \mathrm{H},{ }^{3} J=8.8\right.$ $\mathrm{Hz}), 3.31\left(\mathrm{t}, 4 \mathrm{H},{ }^{3} J=7.7 \mathrm{~Hz}\right), 1.65-1.55(\mathrm{~m}, 4 \mathrm{H}), 1.44-1.31(\mathrm{~m}, 4 \mathrm{H}), 0.98\left(\mathrm{t}, 6 \mathrm{H},{ }^{3} J=7.2\right.$ $\mathrm{Hz}) ;{ }^{13} \mathrm{C} \mathrm{NMR}\left(\mathrm{CDCl}_{3}, 75 \mathrm{MHz}\right): \delta 156.6,156.3,155.8,149.2,148.7,139.3,137.0,134.4$, $133.6,129.2,128.8,127.1,124.0,121.8,121.5,121.1,111.4,107.8,96.1(\mathrm{C} \equiv \mathrm{C}), 86.0(\mathrm{C} \equiv \mathrm{C})$,

50.9, 29.5, 20.5, 14.1; UV-Vis $\left(\mathrm{CH}_{2} \mathrm{Cl}_{2}\right) \lambda \mathrm{nm}\left(\varepsilon, \mathrm{M}^{-1} \mathrm{~cm}^{-1}\right)=378$ (42000), 310sh (19500), 286 (30000), 258sh (32600), 245 (37000); IR (KBr, cm-1) v = 3060 (w), 2955 (m), 2929 (m),

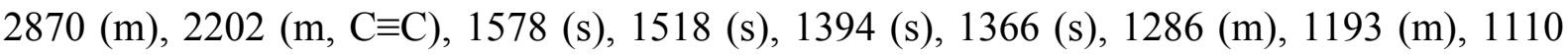
(m), $915(\mathrm{~m}), 812(\mathrm{~m}), 772(\mathrm{~m}), 691(\mathrm{~m}) ; \mathrm{FAB}^{+} \mathrm{m} / \mathrm{z}$ (nature of the peak, relative intensity) $460.2\left([\mathrm{M}+\mathrm{H}]^{+}, 100\right)$; Anal. Calcd for $\mathrm{C}_{32} \mathrm{H}_{33} \mathrm{~N}_{3}: \mathrm{C}, 83.62 ; \mathrm{H}, 7.24 ; \mathrm{N}, 9.14$; Found: $\mathrm{C}, 83.35$; H, 6.99; N, 8.83.

\section{4-(2-(4,6-dimethoxy-1,3,5-triazine)ethynyl)-6-phenyl-2,2'-bipyridine (22). Prepared} using procedure 2; from 4-iodo-6-phenyl-2,2'-bipyridine (100 mg, $0.28 \mathrm{mmol})$, 2-ethynyl-4,6dimethoxy-1,3,5-triazine (60 mg, $0.36 \mathrm{mmol}), \mathrm{Pd}\left(\mathrm{PPh}_{3}\right)_{2} \mathrm{Cl}_{2}(50 \mathrm{mg}, 0.07 \mathrm{mmol}), \mathrm{CuI}(6 \mathrm{mg}$, $0.03 \mathrm{mmol})$, THF $(10 \mathrm{ml})$ and $\mathrm{Et}_{3} \mathrm{~N}(1 \mathrm{ml})$; chromatography on aluminium oxide eluting with 
dichloromethane - petroleum ether ( $v / v 25 / 75$ to 50/50) to give $43 \mathrm{mg}(39 \%)$ of 22 as a white powder after methanol washing; ${ }^{1} \mathrm{H} \mathrm{NMR}\left(\mathrm{CDCl}_{3}, 300 \mathrm{MHz}\right): \delta 8.69(\mathrm{ddd}, 1 \mathrm{H}, J=4.7 \mathrm{~Hz}$, $1.7 \mathrm{~Hz}, 0.8 \mathrm{~Hz}), 8.62-8.59(\mathrm{~m}, 2 \mathrm{H}), 8.15-8.11(\mathrm{~m}, 2 \mathrm{H}), 7.95\left(\mathrm{~d}, 1 \mathrm{H},{ }^{4} J=1.1 \mathrm{~Hz}\right), 7.84(\mathrm{td}$, $\left.1 \mathrm{H},{ }^{3} J=7.7 \mathrm{~Hz},{ }^{4} J=1.7 \mathrm{~Hz}\right), 7.54-7.43(\mathrm{~m}, 3 \mathrm{H}), 7.33(\mathrm{ddd}, 1 \mathrm{H}, J=7.5 \mathrm{~Hz}, 4.8 \mathrm{~Hz}, 1.2 \mathrm{~Hz})$, $4.08(\mathrm{~s}, 6 \mathrm{H}) ;{ }^{13} \mathrm{C} \mathrm{NMR}\left(\mathrm{CDCl}_{3}, 75 \mathrm{MHz}\right): \delta 172.6,162.5,157.0,156.4,155.4,149.3,138.4$, $137.0,130.5,129.7,129.0,127.1,124.3,122.6,122.1,121.4,89.4(\mathrm{C} \equiv \mathrm{C}), 87.8(\mathrm{C} \equiv \mathrm{C}), 55.8$; UV-Vis $\left(\mathrm{CH}_{2} \mathrm{Cl}_{2}\right) \lambda \mathrm{nm}\left(\varepsilon, \mathrm{M}^{-1} \mathrm{~cm}^{-1}\right)=332$ (7900), 288 (39100), 268 (47400), 249 (44200); IR (KBr, cm-1) v = $3019(\mathrm{w}), 2943(\mathrm{w}), 2229(\mathrm{w}, \mathrm{C} \equiv \mathrm{C}), 1522(\mathrm{~s}), 1467$ (m), $1383(\mathrm{~m}), 1338$ (s), $1235(\mathrm{~m}), 1124(\mathrm{~m}), 1000(\mathrm{~m}), 818(\mathrm{~m}), 775(\mathrm{~m}), 691(\mathrm{~m}) ; \mathrm{FAB}^{+} \mathrm{m} / z$ (nature of the peak, relative intensity) $396.1\left([\mathrm{M}+\mathrm{H}]^{+}, 100\right)$; Anal. Calcd for $\mathrm{C}_{23} \mathrm{H}_{17} \mathrm{~N}_{5} \mathrm{O}_{2}: \mathrm{C}, 69.86 ; \mathrm{H}, 4.33 ; \mathrm{N}$, 17.71; Found: C, 69.73; H, 4.24; N, 17.64.

\section{(2-(3,4,5-tris(dodecyloxy)phenyl)ethynyl)(4-iodo-6-phenyl-2,2'-bipyridine)platinum}

(25). Prepared using procedure 4; from (4-iodo-6-phenyl-2,2'-bipyridine)chloroplatinum 23 (35 mg, $0.06 \mathrm{mmol})$, 3,4,5-tris(dodecyloxy)-5-ethynylbenzene (60 mg, $0.09 \mathrm{mmol}), \mathrm{CuI}$ (1 $\left.\mathrm{mg}, 5.10^{-3} \mathrm{mmol}\right)$, DMF $(6 \mathrm{ml})$, dichloromethane $(6 \mathrm{ml})$ and triethylamine $(2 \mathrm{ml})$; chromatography on silica gel eluting with dichloromethane - petroleum ether $(v / v 25 / 75$ to 60/40) to give $46 \mathrm{mg}(64 \%)$ of 25 as a dark red residue; ${ }^{1} \mathrm{H}$ NMR $\left(\mathrm{CDCl}_{3}, 400 \mathrm{MHz}\right): \delta 8.96$ $\left(\mathrm{d}, 1 \mathrm{H},{ }^{3} J=5.5 \mathrm{~Hz}\right), 7.93\left(\mathrm{td}, 1 \mathrm{H},{ }^{3} J=7.9 \mathrm{~Hz},{ }^{4} J=1.7 \mathrm{~Hz}\right), 7.80-7.77(\mathrm{~m}, 2 \mathrm{H}), 7.71(\mathrm{~d}, 1 \mathrm{H}$, $\left.{ }^{3} J=8.0 \mathrm{~Hz}\right), 7.63\left(\mathrm{~d}, 1 \mathrm{H},{ }^{4} J=1.0 \mathrm{~Hz}\right), 7.45-7.41(\mathrm{~m}, 1 \mathrm{H}), 7.17-7.13(\mathrm{~m}, 2 \mathrm{H}), 7.02(\mathrm{td}, 1 \mathrm{H}$, $\left.{ }^{3} J=7.5 \mathrm{~Hz},{ }^{4} J=1.0 \mathrm{~Hz}\right), 6.78(\mathrm{~s}, 2 \mathrm{H}), 4.01\left(\mathrm{t}, 4 \mathrm{H},{ }^{3} J=6.5 \mathrm{~Hz}\right), 3.97\left(\mathrm{t}, 2 \mathrm{H},{ }^{3} J=6.5 \mathrm{~Hz}\right), 1.85$ $-1.73(\mathrm{~m}, 6 \mathrm{H}), 1.53-1.45(\mathrm{~m}, 6 \mathrm{H}), 1.37-1.26(\mathrm{~m}, 48 \mathrm{H}), 0.90-0.86(\mathrm{~m}, 9 \mathrm{H}) ;{ }^{13} \mathrm{C} \mathrm{NMR}$ $\left(\mathrm{CDCl}_{3}, 100 \mathrm{MHz}\right): \delta 165.5,156.9,155.0,152.8,151.7,145.6,143.0,138.8,138.4,137.2$, $131.8,127.7,127.5,127.0,124.8,123.8,123.7,123.2,110.7,106.7,103.8,73.7,69.3,32.1$, 30.5, 29.9, 29.8, 29.7, 29.6, 29.5, 26.4, 22.8, 14.3; UV-Vis $\left(\mathrm{CH}_{2} \mathrm{Cl}_{2}\right) \lambda \mathrm{nm}\left(\varepsilon, \mathrm{M}^{-1} \mathrm{~cm}^{-1}\right)=442$ 
(4000), 370sh (8100), 338 (12700), 283 (40200); IR (KBr, cm-1) v = 3056 (w), 2920 (s), 2852

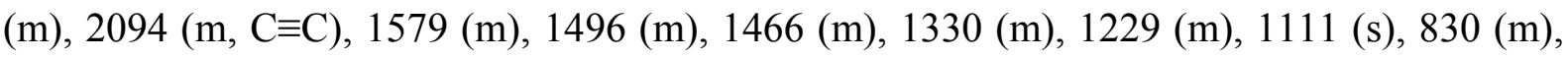
$770(\mathrm{~m}), 722(\mathrm{~m}) ; \mathrm{FAB}^{+} \mathrm{m} / \mathrm{z}$ (nature of the peak, relative intensity) 1208.0, 1207.0, 1206.0

$\left([\mathrm{M}+\mathrm{H}]^{+}, 100,100,60\right)$; Anal. Calcd for $\mathrm{C}_{60} \mathrm{H}_{87} \mathrm{IN}_{2} \mathrm{O}_{3}$ Pt: C, 59.74; H, 7.27; N, 2.32; Found: C, 59.66; H, 7.00; N, 1.99 .

\section{X-ray crystallographic data:}

Compound 2a : CCDC 660943 Formula: $\mathrm{C}_{23} \mathrm{H}_{18} \mathrm{~N}_{2}$. Unit cell parameters: a 9.704(5), b 10.628(5), c 18.515(5), alpha 104.398(5), beta 99.597(5), gamma 101.921(5). Space group P1.

Compound 2b : CCDC 660942 Formula: $\mathrm{C}_{23} \mathrm{H}_{18} \mathrm{~N}_{2}$. Unit cell parameters: a 11.3060(10), b 7.3740(10), c 41.368(5), beta 92.870(2) space group P2 $1 / \mathrm{c}$.

Compound 13 : CCDC 649879. Formula: $\mathrm{C}_{44} \mathrm{H}_{54} \mathrm{~N}_{4}$. Unit cell parameters: a 15.161(5), b 25.720(5), c 10.503(5), beta 107.245(5). Space group $\mathrm{P} 2{ }_{1} / \mathrm{c}$.

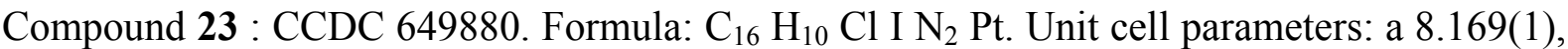
b 12.543(2), c 14.364(2), beta 90.00. Space group Pna2 ${ }_{1}$.

Compound 24 : CCDC 649881. Formula: $\mathrm{C}_{25} \mathrm{H}_{17}$ I N 2 Pt. Unit cell parameters: a 22.190(2), b 16.363(1), c 13.666(1), beta 122.334(2). Space group C2/c.

Compound 26 : CCDC 649882 Formula: $\mathrm{C}_{34} \mathrm{H}_{24} \mathrm{~N}_{2}$ Pt. Unit cell parameters: a 9.701(1), b 16.628(2), c 17.579(2), alpha 67.085(3), beta 89.414(2), gamma 80.768(2). Space group P-1. 
Spectroscopic Data:
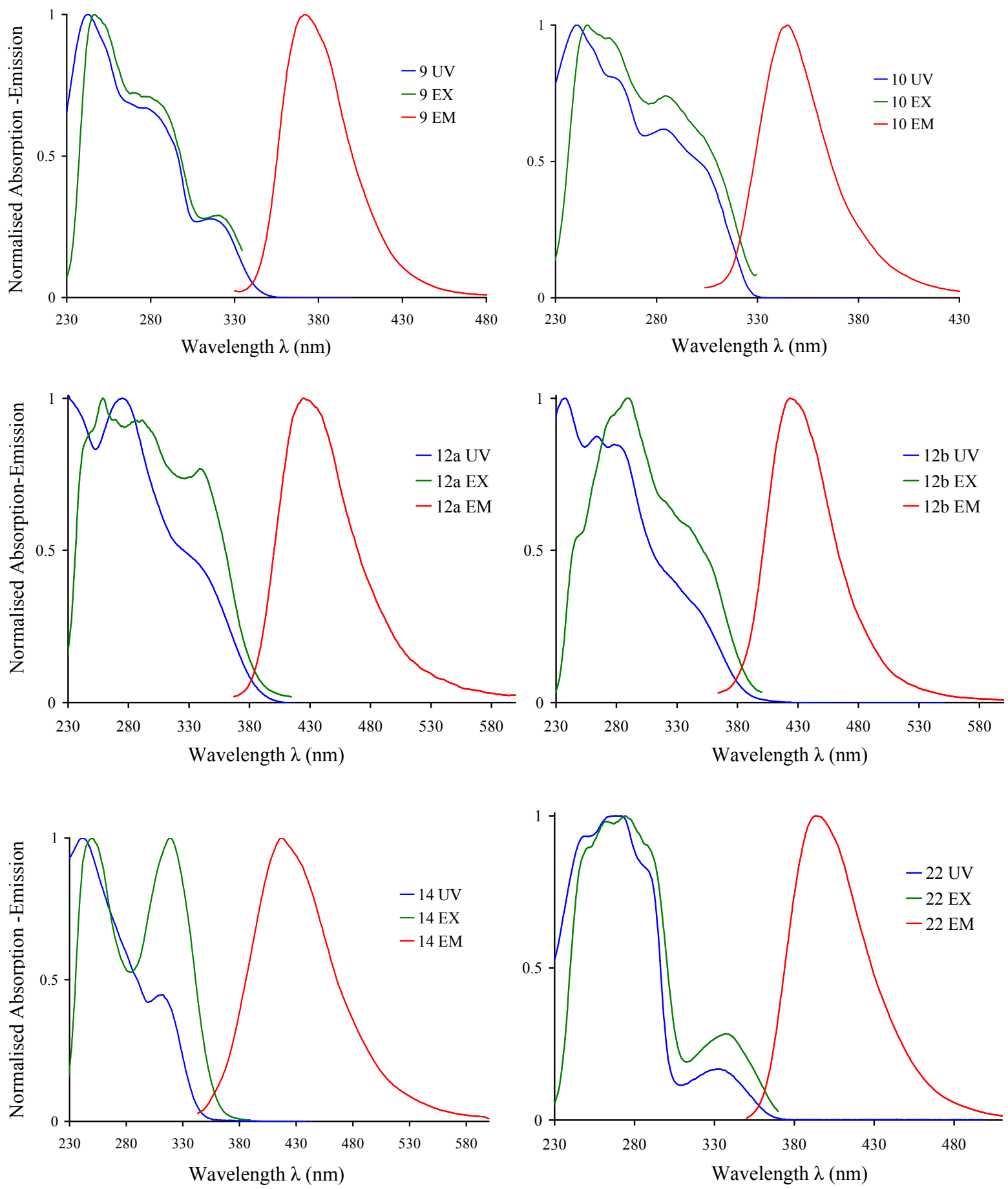

Figure S1. Normalized absorption (blue), excitation (green) and emission (red) spectra of compounds $9,10,12 \mathrm{a}, 12 \mathrm{~b}, 14$ and 22, measured in aerated dichloromethane. 

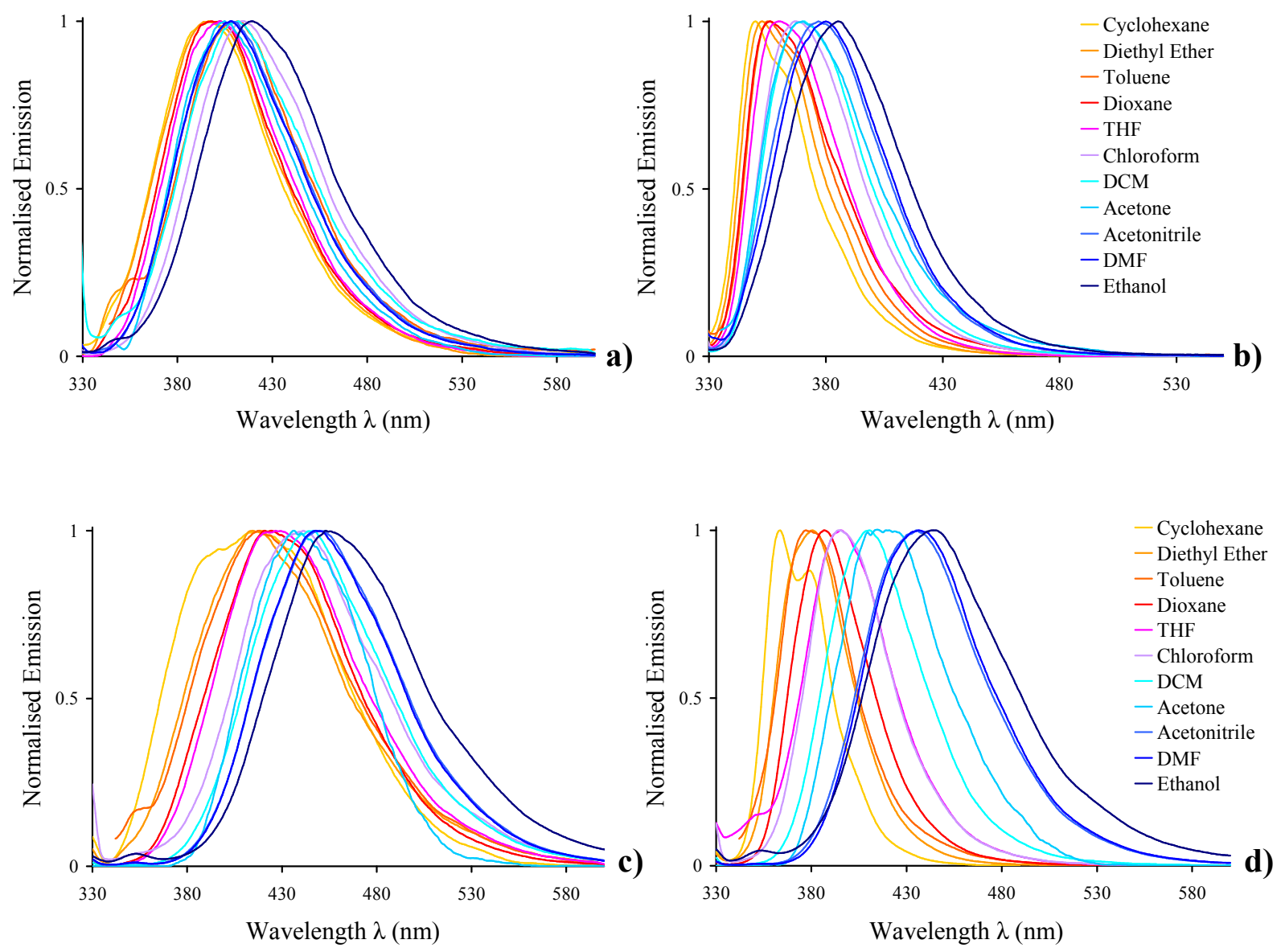

Figure S2. Normalized emission spectra in several solvents of a) $\mathbf{4 a}$; b) $\mathbf{4 b}$; c) 5a; d) $\mathbf{5 b}$.

To further confirm the involvement of CT states, the influence of solvent was studied with the fluorene and carbazole series of compounds. As shown in Figure S2, the fluorescence profile becomes more structured and undergoes a blue shift with decreasing polarity of the solvent. This is particularly noticeable in the carbazole case $\mathbf{5 b}$. Such behavior has been noted for other aromatic compounds and is attributed to close interaction of $\pi, \pi^{*}$ and charge transfer excited singlet states. In addition, in cyclohexane, a vibronic sequence could be seen (Figure 8), where the 0,$0 ; 0,1 ; 0,2 ; 0,3$ transitions in the fluorescence spectrum are separated by $c a$ $1070 \mathrm{~cm}^{-1}$ for $\mathbf{4 b}$ and $1150 \mathrm{~cm}^{-1}$ for $\mathbf{5 b}$. Such vibrational fine structure is typical of the molecular $\mathrm{C}=\mathrm{C}$ frame of bipyridine. 
S18

\section{References supporting information}

(S1) Lipset, F. R. Prog. Dielectr. 1967, 7, 217.

(S2) Olmsted, J. III, J. Phys. Chem. 1979, 83, 2581.

(S3) Dangles, O.; Guibe, F.; Balavoine, G. J. Org. Chem. 1987, 52, 4984.

(S4) Coulson, D. R. Inorg. Synth. 1972, 13, 121. 